International Journal of Pure and Applied Mathematics

Volume 105 No. 3 2015, 459-475

ISSN: 1311-8080 (printed version); ISSN: 1314-3395 (on-line version)

url: http://www.ijpam.eu

doi: http://dx.doi.org/10.12732/ijpam.v105i3.14

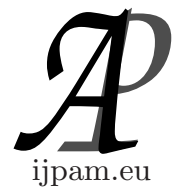

\title{
A NEW APPROACH TO GROUP THEORY VIA SOFT SETS AND L-FUZZY SOFT SETS
}

\author{
Yıldıray Çelik \\ Department of Mathematics \\ Ordu University \\ 52200, Ordu, TURKEY
}

\begin{abstract}
This paper aims to extend the notion of group to inside the algebraic structures of $L$-fuzzy soft sets. We firstly give some new notions such as product, extended product, restricted product of two $L$-fuzzy soft sets. By using these new notions we then introduce concept of $L$-fuzzy soft groups and study some of their properties. We also compare $L$-fuzzy soft groups to the related concept of soft groups. Furthermore, we show that $L$-fuzzy soft groups are more general concept than soft groups. We finally define $L$-fuzzy soft function and $L$-fuzzy soft group homomorphism, and then give theorem of homomorphic image and homomorphic pre-image under a $L$-fuzzy soft function.
\end{abstract}

AMS Subject Classification: 08A72, 20N25

Key Words: $\quad L$-fuzzy subsets, soft sets, soft groups, $L$-fuzzy soft sets, $L$-fuzzy soft groups

\section{Introduction}

To solve complicated problems in economics, engineering, environmental science and social science, methods in classical mathematics are not always successful because of various types of uncertainties present in these problems. While probability theory, fuzzy set theory [20], rough set theory [18], and other mathematical tools are well-known and often useful approaches to describing uncertainty, each of these theories has its inherent difficulties as pointed out in [16]. In 1999, Molodtsov [16] introduced the concept of soft sets, which can be seen as a new

Received: October 2, 2015

(C) 2015 Academic Publications, Ltd. url: www.acadpubl.eu 
mathematical tool for dealing with uncertainties. This so- called soft set theory is free from the difficulties affecting the existing methods.

At present, works on the soft set theory are making progress rapidly. Maji et al. [14] studied several operations on the theory of soft sets. Ali et al. [3] gave some new notions such as restricted intersection, restricted union, restricted difference and extended intersection of two soft sets. Some researches have studied algebraic properties of soft sets. Initially, Aktaş and Çă̆man [2] compared soft sets to the related concepts of fuzzy sets and rough sets. They also defined the notion of soft groups and derived their basic properties using Molodtsov's definition of the soft sets. Feng et al. [6] introduced the notions of soft semirings, soft ideals and idealistic soft semirings, and then investigated several related properties. Kazanci et al. [12] defined soft $B C H$-algebras and gave the theorems of homomorphic image and homomorphic pre-image of soft sets. Acar et al. [1] defined soft rings, and introduced basic notions of soft rings. Çelik et al. [5] defined some new binary relation on soft sets, and also they investigated some new properties of soft rings. Yamak et al. [19] introduced the notion of soft hypergroupoids.

Some researches have studied algebraic properties of fuzzy soft sets. Firstly, Maji et al. [13] defined fuzzy soft set, and established some results on them. Jin-liang et al. [10] defined the operations on fuzzy soft groups, and proved some results on them. Aygünoğlu and Aygün [4] gave the concept of fuzzy soft group, and defined fuzzy soft function and fuzzy soft homomorphism. Majumdar and Samanta [15] defined generalised fuzzy soft sets, and studied some of their properties. They also showed applications of generalised fuzzy soft sets. Jun et al. [11] derived the notion of fuzzy soft BCK/BCI algebras, and investigated its properties. Jiang et al. [9] proposed the notion of the interval-valued intuitionistic fuzzy soft set theory. Lastly, Inan and Öztürk [7] introduced the fuzzy soft ring, and $(\in, \in \vee q)$-fuzzy soft subring. They also studied some of their basic properties.

In this paper, we introduce to group theory via soft sets and $L$-fuzzy soft sets.

The rest of this paper is organized as follows. In Section 2, we give the concepts of $L$-fuzzy subset, soft set, soft group and $L$-fuzzy soft set which will be used throughout the paper. We give some new notions such as product, extended product, restricted product of two $L$-fuzzy soft sets. In Section 3, we introduce the notion of $L$-fuzzy soft group and study its characteristic properties. We then give some related examples. We also investigated relations between soft groups and $L$-fuzzy soft groups. Furthermore, we show that $L$ fuzzy soft groups are more general concept than soft groups. Lastly, we define 
$L$-fuzzy soft function and $L$-fuzzy soft group homomorphism, and then give theorem of homomorphic image and homomorphic pre-image under a $L$-fuzzy soft function.

\section{Preliminaries}

In this section, we will give some known and useful definitions and notations regarding $L$-fuzzy subset, $L$-fuzzy subgrop, soft set, soft group, $L$-fuzzy soft set.

\subsection{L-Fuzzy Subsets on Groups}

The definitions and notions, in this part, may be found in references ([8], [17], $[20])$.

Throughout this paper, let $(G, \cdot)$ be a group, and $L$ be a complete lattice. A non-empty subset $H$ of a group $G$ is called a subgroup, is denoted $H \prec G$, if and only if $a \cdot b^{-1}$ for all $a, b \in G$. The subgroups $\left\{e_{G}\right\}$ and $G$ are called trivial subgroups of $G$, where $e_{G}$ is unit element of $G$. The set of all subgroups of $G$ is denoted $S(G)$.

Let $G$ and $H$ be two groups. A mapping $f: G \rightarrow H$ is called a homomorphism of groups if it satisfies

$$
f(a \cdot b)=f(a) \cdot f(b)
$$

for all $a, b \in G$. That is, the mapping $f$ preserves the group operations. A group homomorphism $f: G \rightarrow H$ is called a monomorphism [resp. epimorphism, isomorphism] if it is an injective [resp. surjective, bijective] mapping. The sets Ker $f=\left\{g \in G \mid f(g)=e_{H}\right\}$ and $\operatorname{Im} f=\{h \in H \mid f(g)=h$ for some $g \in G\}$ are called kernel and image of $f$, respectively.

A $L$-fuzzy subset $\mu$ of $G$ is defined as a map from $G$ to $L$. If $L=[0,1]$, then $L$-fuzzy subset is called fuzzy subset. The family of all $L$-fuzzy subsets of $G$ is denoted by $\mathcal{F} L(G)$. The following are most popular operations on $L$-fuzzy subsets:

$$
\forall \mu, \nu \in \mathcal{F} L(G), \quad \omega \in \mathcal{F} L(H)
$$

and $x \in G, y \in H$;

$$
\begin{aligned}
(\mu \vee \nu)(x) & =\mu(x) \vee \nu(x) \\
(\mu \wedge \nu)(x) & =\mu(x) \wedge \nu(x) \\
(\mu \times \omega)(x, y) & =\mu(x) \wedge \omega(y)
\end{aligned}
$$




$$
\begin{aligned}
(\mu \cdot \nu)(x) & =\bigvee_{x=a \cdot b}(\mu(a) \wedge \nu(b)) ; \\
\mu^{-1}(x) & =\mu\left(x^{-1}\right) ; \\
f(\mu)(y) & = \begin{cases}\bigvee_{f(a)=y} \mu(a) & \text { if } f^{-1}(y) \neq \emptyset \\
0 & \text { otherwise. }\end{cases} \\
f^{-1}(\omega)(x) & =\omega(f(x))
\end{aligned}
$$

$\mu \leq \nu$ if and only if $\mu(x) \leq \nu(x)$ for all $x \in G$. For $T \subseteq G, \chi_{T} \in \mathcal{F} L(G)$ is called characteristic function of $T$, and defined by $\chi_{T}(x)=1$ if $x \in T$ and $\chi_{T}(x)=0$ otherwise for all $x \in G$.

For any $\mu \in \mathcal{F} L(G)$, the set $\{x \mid x \in G, \mu(x) \geq \alpha\}$ is called $\alpha$-level subset of $\mu$ and denoted by $\mu_{\alpha}$.

Theorem 1. [17] Let $\mu, \nu \in \mathcal{F} L(G)$, and $L$ be a lattice. Then the following statements hold:

(1) If $\mu \leq \nu$ and $\alpha \in L$, then $\mu_{\alpha} \subseteq \nu_{\alpha}$

(2) If $\alpha \leq \beta$ and $\alpha, \beta \in L$, then $\mu_{\beta} \subseteq \nu_{\alpha}$

(3) $\mu=\nu$ if and only if $\mu_{\alpha}=\nu_{\alpha}$ for all $\alpha \in L$

(4) $\mu_{\alpha} \vee \nu_{\alpha} \subseteq(\mu \vee \nu)_{\alpha}$ for all $\alpha \in L$

(5) $\mu_{\alpha} \wedge \nu_{\alpha}=(\mu \wedge \nu)_{\alpha}$ for all $\alpha \in L$

Definition 2. [17] Let $\mu \in \mathcal{F} L(G)$. Then $\mu$ is called a $L$-fuzzy subgroup of $G$, and is denoted $\mu \in \mathcal{F} L_{S}(G)$ if

(i) $\mu(a b) \geq \mu(a) \wedge \mu(b)$

(ii) $\mu\left(a^{-1}\right) \geq \mu(a)$

for all $a, b \in G$.

Theorem 3. [17] Let $f: G \rightarrow H$ be a group homomorphism. Let $\mu \in$ $\mathcal{F} L_{S}(G)$ and $\nu \in \mathcal{F} L_{S}(H)$. Then $f(\mu) \in \mathcal{F} L_{S}(H)$ and $f^{-1}(\nu) \in \mathcal{F} L_{S}(G)$, respectively.

Theorem 4. [17] Let $\oslash \neq H \subseteq G$. Then $\chi_{H}$ is $L$-fuzzy subgroup of $G$ if and only if $H \prec G$.

Theorem 5. [17] Let $\mu$ and $\nu$ be two L-fuzzy subgroups of $G$. Then $\mu \cdot \nu$ is $L$-fuzzy subgroup of $G$ if and only if $\mu \cdot \nu=\nu \cdot \mu$. 


\subsection{Soft Sets and Soft Groups}

Molodtsov [16] defined the notion of a soft set in the following way: Let $G$ be an inital universe set and $E$ be a set of parameters. The power set of $G$ is denoted by $\mathcal{P}(G)$ and $A$ is a subset of $E$. A pair $(F, A)$ is called a soft set over $G$, where $F$ is a mapping given by $F: A \rightarrow \mathcal{P}(G)$. In other words, a soft set over $G$ is a parameterized family of subsets of the universe $G$. For $x \in A, F(x)$ may be considered as the set of $x$-approximate elements of the soft set $(F, A)$.

Definition 6. [16] Let $(F, A)$ and $(K, B)$ be two soft sets over $G$. Then, $(F, A)$ is said to be a soft subset of $(K, B)$, denoted $(F, A) \subseteq(K, B)$, if $A \subseteq B$ and $F(a) \subseteq K(a)$ for all $a \in A$.

Definition 7. ([5],[6]) Let $(F, A)$ and $(K, B)$ be two soft sets over $G$. Then,

(1) The restricted intersection of soft sets $(F, A)$ and $(K, B)$ is defined as the soft set $(N, C)=(F, A) \cap(K, B)$ over $G$, where $C=A \cap B$, and $N(c)=F(c) \cap K(c)$ for all $c \in C$.

(2) The extended union of soft sets $(F, A)$ and $(K, B)$ is defined as the soft set $(N, C)=(F, A) \cup(K, B)$ over $G$, where $C=A \cup B$, and

$$
N(c)=\left\{\begin{array}{lll}
F(c) & \text { if } & c \in A \backslash B \\
K(c) & \text { if } & c \in B \backslash A \\
F(c) \cup K(c) & \text { if } & c \in A \cap B
\end{array}\right.
$$

for all $c \in C$.

Definition 8. [2] Let $(F, A)$ be soft set over a group $G$. Then $(F, A)$ is called a soft group over $G$ if $F(a) \prec G$ for all $a \in A$. The family of all soft groups over $G$ is denoted by $\mathcal{U}$.

Definition 9. Let $\mu \in \mathcal{F} L(G)$ and $\mu_{\alpha}$ be $\alpha$-level set of $\mu$. Then the soft set $\left(F_{\mu}, L\right)$, where $F_{\mu}: L \rightarrow \mathcal{P}(G)$, defined by $F_{\mu}(\alpha)=\mu_{\alpha}$ for all $\alpha \in L$, is called level soft set of $\mu$ over $G$.

If $L$ is choosen closed unit interval $[0,1]$, then Definition 9 coincides with [2].

Theorem 10. Let $\mu, \nu \in \mathcal{F} L(G)$. Let $\left(F_{\mu}, L\right)$ and $\left(F_{\nu}, L\right)$ be two level soft sets over $G$. Then,

(1) If $\mu \leq \nu$, then $\left(F_{\mu}, L\right) \subseteq\left(F_{\nu}, L\right)$

(2) $\left(F_{\mu}, L\right) \cup\left(F_{\nu}, L\right) \subseteq\left(F_{\mu \vee \nu}, L\right)$. 
(3) $\left(F_{\mu}, L\right) \cap\left(F_{\nu}, L\right)=\left(F_{\mu \wedge \nu}, L\right)$

(4) If $L$ is chain, then $\left(F_{\mu}, L\right) \cup\left(F_{\nu}, L\right)=\left(F_{\mu \vee \nu}, L\right)$.

Proof. By Theorem 1, the proof can be achieved.

Theorem 11. (1) The mapping $\gamma: S(G) \rightarrow \mathcal{F} L(G)$, defined by $\gamma(H)=$ $\chi_{H}$ for all $H \in S(G)$, is a lattice monomorphism.

(2) The mapping $\gamma: \mathcal{F} L(G) \rightarrow \mathcal{U}$, defined by $\gamma(\mu)=\left(F_{\mu}, L\right)$ for all $\mu \in$ $\mathcal{F} L(G)$, is a one-to-one and increasing function. If $L$ is chain, then $\gamma$ is lattice homomorphism.

Proof.

(1) Let $H_{1}, H_{2} \in S(G)$ and $\gamma\left(H_{1}\right)=\gamma\left(H_{2}\right)$. Let $x \in H_{1}$ for all $x \in G$. Since $1=\chi_{H_{1}}(x)=\chi_{H_{2}}(x)$, then $x \in H_{2}$. So $H_{1} \subseteq H_{2}$. Similarly $H_{2} \subseteq H_{1}$. Hence $H_{1}=H_{2}$. Furthermore $\gamma$ is monomorphism. Clearly we can see $\gamma\left(H_{1} \cap H_{2}\right)=\gamma\left(H_{1}\right) \wedge \gamma\left(H_{2}\right)$ and $\gamma\left(H_{1} \cup H_{2}\right)=\gamma\left(H_{1}\right) \vee \gamma\left(H_{2}\right)$. Cosequently, the mapping $\gamma$ is lattice monomorphism.

(2) By Theorem $1(1,3), F_{\mu}(\alpha)=\mu_{\alpha}$ for all $\alpha \in L$, is one-to-one and increasing mapping. If the lattice $L$ is chain, then $\gamma$ is lattice homomorphism by Theorem 10(4).

Corollary 12. By Theorem 11(1), the subgroups of $G$ can be embedded into L-fuzzy subgroups of $G$. By Theorem 11(2), If $L$ is chain, then $L$-fuzzy sublattice of $G$ can be taken $L$-parameter soft group.

On the other words, soft groups are more general concept than subgroups and $L$-fuzzy subgroups.

\subsection{L-Fuzzy Soft Sets and Operations}

In this part, we define the concept of $L$-fuzzy soft sets, and give some notations regarding of them.

Definition 13. Let $G$ be a common universe, $E$ be a set of parameters and $A \subseteq E$. Then a pair $(F, A)$ is called a $L$-fuzzy soft set over $G$, where $F$ is a mapping given by $F: A \rightarrow \mathcal{F} L(G)$.

Example 1. Let $G=\left\{h_{1}, h_{2}, h_{3}, h_{4}\right\}$ be the set of four houses under consideration, $E=\left\{e_{1}\right.$ (costly), $e_{2}$ (beautiful),$e_{3}$ (green surroundings), $e_{4}$ (wooden), $e_{5}$ (luxurious 
be the set of parameters and $A=\left\{e_{1}, e_{2}, e_{3}\right\} \subset E$. Let $\alpha_{i} \in L, i \in\{1,2, \ldots, 9\}$, where $\alpha_{i} \leq \alpha_{j}$ for all $i \leq j$. Then

$$
\begin{aligned}
& (F, A)=\left\{\left\{\quad F\left(e_{1}\right)=\left\{h_{1} / \alpha_{8}, h_{2} / \alpha_{2}, h_{3} / \alpha_{3}, h_{4} / \alpha_{7}\right\},\right.\right. \\
& F\left(e_{2}\right)=\left\{h_{1} / \alpha_{9}, h_{2} / \alpha_{7}, h_{3} / \alpha_{2}, h_{4} / \alpha_{6}\right\}, \\
& \left.F\left(e_{3}\right)=\left\{h_{1} / \alpha_{2}, h_{2} / \alpha_{3}, h_{3} / \alpha_{8}, h_{4} / \alpha_{4}\right\}\right\}
\end{aligned}
$$

is the $L$-fuzzy soft set representing the 'attractiveness of the house' which Mr. $\mathrm{X}$ is going to buy.

Definition 14. For two $L$-fuzzy soft sets $(F, A)$ and $(K, B)$ over a common universe $G$, we say that $(F, A)$ is a $L$-fuzzy soft subset of $(K, B)$ if

(i) $A \subseteq B$

(ii) $F(a) \leq K(a)$ for all $a \in A$. In this case, we write $(F, A) \widetilde{\widetilde{C}}(K, B)$.

Definition 15. Let $(F, A)$ and $(K, B)$ be two $L$-fuzzy soft sets over $G$. Then,

(1) The extended union of $L$-fuzzy soft sets $(F, A)$ and $(K, B)$ is defined as the $L$-fuzzy soft set $(N, C)=(F, A) \widetilde{\cup}(K, B)$ over $G$, where $C=A \cup B$ and

$$
N(c)=\left\{\begin{array}{lll}
F(c) & \text { if } & c \in A \backslash B \\
K(c) & \text { if } & c \in B \backslash A \\
F(c) \vee K(c) & \text { if } & c \in A \cap B
\end{array}\right.
$$

for all $c \in C$.

(2) The restricted union of $L$-fuzzy soft sets $(F, A)$ and $(K, B)$ is defined as the $L$-fuzzy soft set $(N, C)=(F, A) \widetilde{\cup}_{\Re}(K, B)$ over $G$, where $C=A \cap B$, and $N(c)=F(c) \vee K(c)$ for all $c \in C$.

(3) The extenden intersection of $L$-fuzzy soft sets $(F, A)$ and $(K, B)$ is defined as the $L$-fuzzy soft set $(N, C)=(F, A) \widetilde{\cap}_{\varepsilon}(K, B)$ over $G$, where $C=A \cup B$, and

$$
N(c)=\left\{\begin{array}{lll}
F(c) & \text { if } & c \in A \backslash B \\
K(c) & \text { if } & c \in B \backslash A \\
F(c) \wedge K(c) & \text { if } & c \in A \cap B
\end{array}\right.
$$

for all $c \in C$. 
(4) The restricted intersection of $L$-fuzzy soft sets $(F, A)$ and $(K, B)$ is defined as the $L$-fuzzy soft set $(N, C)=(F, A) \widetilde{\cap}(K, B)$ over $G$, where $C=A \cap B$, and $N(c)=F(c) \wedge K(c)$ for all $c \in C$.

(5) The $\wedge$-intersection of $L$-fuzzy soft sets $(F, A)$ and $(K, B)$ is defined as the $L$-fuzzy soft set $(N, C)=(F, A) \widetilde{\wedge}(K, B)$ over $G$, where $C=A \times B$, and $N(a, b)=F(a) \wedge K(b)$ for all $(a, b) \in A \times B$.

(6) The $\vee$-union of $L$-fuzzy soft sets $(F, A)$ and $(K, B)$ is defined as the $L$ fuzzy soft set $(N, C)=(F, A) \widetilde{V}(K, B)$ over $G$, where $C=A \times B$, and $N(a, b)=F(a) \vee K(b)$ for all $(a, b) \in A \times B$.

(7) The cartesian product of $L$-fuzzy soft sets $(F, A)$ and $(K, B)$ is defined as the $L$-fuzzy soft set $(N, C)=(F, A) \widetilde{\times}(K, B)$ over $G$, where $C=A \times B$, and $N(a, b)=F(a) \times K(b)$ for all $(a, b) \in A \times B$.

(8) The product of $L$-fuzzy soft sets $(F, A)$ and $(K, B)$ is defined as the $L$ fuzzy soft set $(N, C)=(F, A) \widetilde{\odot}(K, B)$ over $G$, where $C=A \times B$, and $N(a, b)=F(a) \cdot K(b)$ for all $(a, b) \in A \times B$.

(9) The extended product of $L$-fuzzy soft sets $(F, A)$ and $(K, B)$ is defined as the $L$-fuzzy soft set $(N, C)=(F, A) \widetilde{\odot}(K, B)$ over $G$, where $C=A \cup B$, and

$$
N(c)=\left\{\begin{array}{lll}
F(c) & \text { if } & c \in A \backslash B \\
K(c) & \text { if } & c \in B \backslash A \\
F(c) \cdot K(c) & \text { if } & c \in A \cap B
\end{array}\right.
$$

for all $c \in C$.

(10) The restricted product of $L$-fuzzy soft sets $(F, A)$ and $(K, B)$ is defined as the $L$-fuzzy soft set $(N, C)=(F, A) \widetilde{\cap}(K, B)$ over $G$, where $C=A \cap B$, and $N(c)=F(c) \cdot K(c)$ for all $c \in C$.

(11) The inverse of $L$-fuzzy soft set $(F, A)$ is defined as the $L$-fuzzy soft set $(N, A)=(F, A)^{-1}$ over $G$, where $N(x)=F(x)^{-1}$ for all $x \in A$.

Definition $15(1,5,6)$ were given in [13] for the special cases $L=[0,1]$.

To illustrate above definition, let us consider the following example.

Example 2. Let $G=\mathbb{Z}_{4}, A=\{1,2\}, B=\{2,3\}$ and $L=[0,1]$. Let $(F, A)$ and $(K, B)$ two $L$-fuzzy soft sets over $G$ defined by $F(x)(\bar{n}):=\frac{x}{n+x}$ and $K(x)(\bar{n}):=\frac{n}{n+x}$ for all $\bar{n} \in \mathbb{Z}_{4}$. Then

$$
(F, A) \widetilde{\cup}(K, B)=\left(N_{1}, C\right),
$$


where $C=A \cup B=\{1,2,3\}$, and for all $\bar{n} \in \mathbb{Z}_{4}$

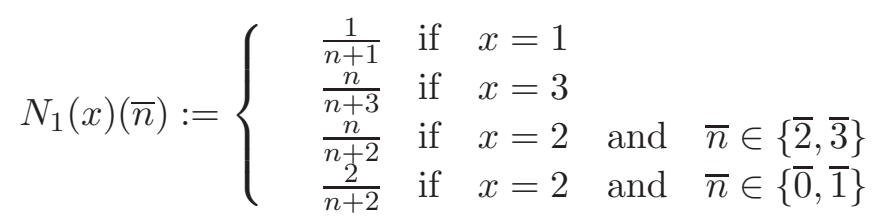

$(F, A) \widetilde{\cup}_{\Re}(K, B)=\left(N_{2}, C\right)$, where $C=A \cap B=\{2\}$, and for all $\bar{n} \in \mathbb{Z}_{4}$

$$
N_{2}(2)(\bar{n}):=\left\{\begin{array}{cll}
\frac{n}{n+2} & \text { if } & \bar{n} \in\{\overline{2}, \overline{3}\} \\
\frac{2}{n+2} & \text { if } & \bar{n} \in\{\overline{0}, \overline{1}\}
\end{array}\right.
$$

$(F, A) \widetilde{\cap}_{\varepsilon}(K, B)=\left(N_{3}, C\right)$, where $C=A \cup B=\{1,2,3\}$, and for all $\bar{n} \in \mathbb{Z}_{4}$

$$
N_{3}(x)(\bar{n}):=\left\{\begin{array}{ccc}
\frac{1}{n+1} & \text { if } x=1 \\
\frac{n}{n+3} & \text { if } x=3 \\
\frac{2}{n+2} & \text { if } x=2 \text { and } \bar{n} \in\{\overline{2}, \overline{3}\} \\
\frac{n}{n+2} & \text { if } x=2 \text { and } \bar{n} \in\{\overline{0}, \overline{1}\}
\end{array}\right.
$$

$(F, A) \widetilde{\cap}(K, B)=\left(N_{4}, C\right)$, where $C=A \cap B=\{2\}$, and for all $\bar{n} \in \mathbb{Z}_{4}$

$$
N_{4}(2)(\bar{n}):=\left\{\begin{array}{cll}
\frac{2}{n+2} & \text { if } & \bar{n} \in\{\overline{2}, \overline{3}\} \\
\frac{n}{n+2} & \text { if } & \bar{n} \in\{\overline{0}, \overline{1}\}
\end{array}\right.
$$

$(F, A) \widetilde{\wedge}(K, B)=\left(N_{5}, C\right)$, where $C=A \times B=\{(1,2),(1,3),(2,2),(2,3)\}$, and for all $\bar{n} \in \mathbb{Z}_{4}$

$$
N_{5}(x, y)(\bar{n}):=\min \left\{\frac{x}{n+x}, \frac{n}{n+y}\right\}=\left\{\begin{array}{cll}
\frac{n}{n+3} & \text { if } & \bar{n} \in\{\overline{0}, \overline{1}\} \\
\frac{1}{n+1} & \text { if } & \bar{n} \in\{\overline{2}, \overline{3}\}
\end{array}\right.
$$

$(F, A) \widetilde{V}(K, B)=\left(N_{6}, C\right)$, where $C=A \times B=\{(1,2),(1,3),(2,2),(2,3)\}$, and for all $\bar{n} \in \mathbb{Z}_{4}$

$$
N_{6}(x, y)(\bar{n}):=\max \left\{\frac{x}{n+x}, \frac{n}{n+y}\right\}=\left\{\begin{array}{cll}
\frac{2}{n+2} & \text { if } & \bar{n} \in\{\overline{0}, \overline{1}\} \\
\frac{n}{n+2} & \text { if } & \bar{n} \in\{\overline{2}, \overline{3}\}
\end{array}\right.
$$

$(F, A) \widetilde{\odot}(K, B)=\left(N_{7}, C\right)$, where $C=A \times B=\{(1,2),(1,3),(2,2),(2,3)\}$, and for all $\bar{n} \in \mathbb{Z}_{4}$

\begin{tabular}{l|cccc}
$N_{7}$ & $\overline{0}$ & $\overline{1}$ & $\overline{2}$ & $\overline{3}$ \\
\hline$N_{7}(1,2)$ & $\frac{1}{2}$ & $\frac{1}{3}$ & $\frac{1}{2}$ & $\frac{3}{5}$ \\
$N_{7}(1,3)$ & $\frac{1}{2}$ & $\frac{1}{3}$ & $\frac{2}{5}$ & $\frac{1}{2}$ \\
$N_{7}(2,2)$ & $\frac{3}{5}$ & $\frac{1}{2}$ & $\frac{1}{2}$ & $\frac{3}{5}$ \\
$N_{7}(2,3)$ & $\frac{1}{2}$ & $\frac{1}{2}$ & $\frac{2}{5}$ & $\frac{3}{5}$
\end{tabular}


$(F, A) \widetilde{\odot} \cup(K, B)=\left(N_{8}, C\right)$, where $C=A \cup B=\{1,2,3\}$, and for all $\bar{n} \in \mathbb{Z}_{4}$

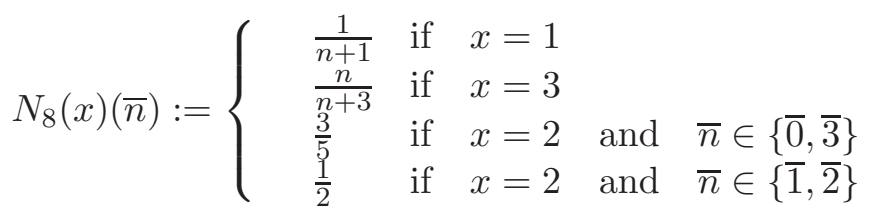

$(F, A) \widetilde{\odot}_{\cap}(K, B)=\left(N_{9}, C\right)$, where $C=A \cap B=\{2\}$, and for all $\bar{n} \in \mathbb{Z}_{4}$

$$
N_{9}(2)(\bar{n}):=\left\{\begin{array}{lll}
\frac{3}{5} & \text { if } & \bar{n} \in\{\overline{0}, \overline{3}\} \\
\frac{1}{2} & \text { if } & \bar{n} \in\{\overline{1}, \overline{2}\}
\end{array}\right.
$$

Now, we can define a genaral binary operation on $L$-fuzzy soft sets in the following way:

Suppose that $\oplus$ is a binary operation on $P(E)$, and $\otimes$ is a binary operation on $\mathcal{F} L(G)$. Then for any two $L$-fuzzy soft set $(F, A)$ and $(K, B)$ over $G$, $(F, A) \oplus_{\otimes}(K, B)$ is defined as the $L$-fuzzy soft set $(N, C)$ where $C=A \oplus B$ and

$$
N(c)= \begin{cases}F(c) & \text { if } \quad c \in A \backslash B \\ K(c) & \text { if } c \in B \backslash A \\ F(c) \otimes K(c) & \text { if } c \in A \cap B \\ 0_{G} & \text { otherwise }\end{cases}
$$

for all $c \in C$. Here we describe a general binary operation on $L$-fuzzy soft sets obtained as a special case of the some binary relations such as extended union, restricted union, extended intersection,restricted intersection, restricted product and extended product for the $\oplus \in\{\cup, \cap\}$ and $\otimes \in\{\vee, \wedge, \cdot\}$.

\section{A New Approach to Group Theory via Soft Sets and $L$-Fuzzy Soft Sets}

The notion of fuzzy soft groups was introduced in ([4], [10]). In this section, we introduce the concept of $L$-fuzzy soft groups which extends the notion of group to include the algebraic structures of $L$-fuzzy soft sets, and give some new fundamental properties of $L$-fuzzy soft groups.

Definition 16. Let $(F, A)$ be a $L$-fuzzy soft set over $G$. Then $(F, A)$ is called a $L$-fuzzy soft group over $G$ if $F(a)$ is a $L$-fuzzy subgroup of $G$ for all $a \in A$. 
Example 3. Let $\mu \in \mathcal{F} L_{S}(G)$. Then the soft set $(H, L)$ over $G$, defined by $H(\alpha)=\chi_{\mu_{\alpha}}$ for all $\alpha \in L$, is a $L$-fuzzy soft group over $G$.

Clearly $\mu_{\alpha} \prec G$. By Theorem $4, \chi_{\mu_{\alpha}}$ is $L$-fuzzy subgroup over $G$. Hence $(H, L)$ is a $L$-fuzzy soft group over $G$.

Example 4. Let $L=\{0, \alpha, \beta, 1\}$ be a lattice, where $0<\alpha<\beta<1$. Let $F: \mathbb{Z}_{2} \times \mathbb{Z}_{2} \rightarrow L^{\mathbb{Z}_{2} \times \mathbb{Z}_{2}}$ be a set-valued mapping defined by

$F(\bar{x}, \bar{y})(\overline{0}, \overline{0})=1, F(\bar{x}, \bar{y})(\overline{1}, \overline{0})=\alpha, F(\bar{x}, \bar{y})(\overline{0}, \overline{1})=F(\bar{x}, \bar{y})(1,1)=\beta$.

Then it is easy to see that the $L$-fuzzy soft set $\left(F, \mathbb{Z}_{2} \times \mathbb{Z}_{2}\right)$ is a $L$-fuzzy soft group over $\mathbb{Z}_{2} \times \mathbb{Z}_{2}$.

Example 5. Let $G$ be a group. Let $(F, \mathbb{N})$ be a $L$-fuzzy soft set over $G$ defined by

$$
F(n)(x)=\left\{\begin{array}{lrr}
1 & \text { if } & x^{n}=e_{G} \\
\alpha & \text { otherwise }
\end{array}\right.
$$

for all $n \in \mathbb{N}, x \in G, \alpha \in L$. Then $(F, \mathbb{N})$ is a $L$-fuzzy soft group over $G$.

Theorem 17. If $(F, A)$ is a soft group over $G$, then the $L$-fuzzy soft set $(\bar{F}, A)$, defined by $\bar{F}(a)=\chi_{F(a)}$ for all $a \in A$, is a $L$-fuzzy soft group over $G$.

Proof. By Theorem 4, the proof can be achieved.

Theorem 18. Let $\mathcal{U}$ and $\tilde{\mathcal{U}}$ be a family of all soft groups and L-fuzzy soft groups over $G$, respectively. Then the mapping $\gamma: \mathcal{U} \rightarrow \widetilde{\mathcal{U}}$, defined by $\gamma(F, L)=(\bar{F}, L)$, is a one-to-one lattice homomorphism.

Proof. From the proof of Theorem 11(2) and by Theorem 17, the proof can be achieved.

Corollary 19. By Theorem 18, a soft group can be embedded into L-fuzzy soft groups.

On the other words, L-fuzzy soft groups are more general concept than soft groups.

Theorem 20. Let $(F, A)$ and $(K, B)$ be two L-fuzzy soft groups over $G$. Then,

(1) $(F, A) \widetilde{\cap}_{\varepsilon}(K, B)$ is a $L$-fuzzy soft group over $G$

(2) $(F, A) \widetilde{\cap}(K, B)$ is a L-fuzzy soft group over $G$

(3) If $F(c) \leq K(c)$ or $K(c) \leq F(c)$ for all $c \in A \cap B$, then $(F, A) \widetilde{\cup}(K, B)$ is a $L$-fuzzy soft group over $G$ 
(4) If $F(c) \leq K(c)$ or $K(c) \leq F(c)$ for all $c \in A \cap B$, then $(F, A) \widetilde{\cup}_{\Re}(K, B)$ is a $L$-fuzzy soft group over $G$

(5) $(F, A) \widetilde{\wedge}(K, B)$ is a L-fuzzy soft group over $G$

(6) If $F(a) \leq K(b)$ or $K(b) \leq F(a)$ for all $(a, b) \in A \times B$, then $(F, A) \widetilde{V}(K, B)$ is a $L$-fuzzy soft group over $G$.

Proof.

(1) Let $(F, A) \widetilde{\cap}_{\varepsilon}(K, B)=(N, A \cup B)$. Clearly, if $c \in A \backslash B$ or $c \in B \backslash A$ for all $c \in A \cup B$, then $N(c)$ is a $L$-fuzzy subgroup of $G$. If $c \in A \cap B$, then $N(c)=F(c) \wedge K(c)$ is a $L$-fuzzy subgroup of $G$ since the intersection of two $L$-fuzzy subgroup is a $L$-fuzzy subgroup over $G$. Hence, $(F, A) \widetilde{\cap}_{\varepsilon}(K, B)$ is a $L$-fuzzy soft group over $G$.

(2) It is similar to the proof of (1)

(3) Let $(F, A) \widetilde{\cup}(K, B)=(N, A \cup B)$. Clearly, if $c \in A \backslash B$ or $c \in B \backslash A$ for all $c \in A \cup B$, then $N(c)$ is a $L$-fuzzy subgroup of $G$. If $F(c) \leq K(c)$ or $K(c) \leq F(c)$, then $N(c)=F(c) \vee K(c)$ is a $L$-fuzzy subgroup of $G$ for all $c \in A \cap B$. Hence, $(F, A) \widetilde{\cup}(K, B)$ is a $L$-fuzzy soft group over $G$.

(4) It is similar to the proof of (3)

(5) Let $(F, A) \widetilde{\wedge}(K, B)=(N, A \times B)$. We know that $F(a)$ and $K(b)$ are $L$ fuzzy subgroups of $G$ for all $a \in A, b \in B$. Since intersection of two $L$-fuzzy subgroups is a $L$-fuzzy subgroup, then $N(a, b)=F(a) \wedge K(b)$ is also $L$-fuzzy subgroup of $G$. Hence $(F, A) \widetilde{\wedge}(K, B)$ is a $L$-fuzzy soft group over $G$.

(6) It is similar to the proof of (3).

Theorem 21. Let $(F, A)$ and $(K, B)$ be two $L$-fuzzy soft groups over $G_{1}$ and $G_{2}$, respectively. Then $(F, A) \widetilde{\times}(K, B)$ is a $L$-fuzzy soft group over $G_{1} \times G_{2}$.

Proof. Let $(F, A) \widetilde{\times}(K, B)=(N, C)$, where $C=A \times B$, and for all $(a, b) \in$ $A \times B$, we have $N(a, b)=F(a) \times K(b)$. Since $(F, A)$ and $(K, B)$ are $L$-fuzzy soft groups over $G_{1}$ and $G_{2}$, then $F(a)$ and $K(b)$ are $L$-fuzzy subgroups of $G_{1}$ and $G_{2}$, respectively, for all $(a, b) \in A \times B$. Also, for all $x \in G_{1}$ and $y \in G_{2}$, we write $(F(a) \times K(b))(x, y)=F(a)(x) \wedge K(b)(y)$. Hence, we obtain $N(a, b)=F(a) \times K(b)$ is a $L$-fuzzy subgroup of $G_{1} \times G_{2}$ for all $(a, b) \in A \times B$. Consequently, $(F, A) \widetilde{\times}(K, B)$ is a $L$-fuzzy soft group over $G_{1} \times G_{2}$. 
Theorem 22. Let $(F, A)$ and $(K, A)$ be two L-fuzzy soft groups over $G$. Then $(F, A) \widetilde{\odot}(K, A)$ is a $L$-fuzzy soft group over $G$ if and only if

$$
(F, A) \widetilde{\odot}(K, A)=(K, A) \widetilde{\odot}(F, A) .
$$

Proof. By Theorem 5, the proof can be achieved.

Theorem 23. Let $(F, A)$ be a $L$-fuzzy soft set over $G$. Then $(F, A)$ is a $L$ fuzzy soft group over $G$ if and only if $(F, A) \widetilde{\odot}_{\cap}(F, A) \widetilde{\subseteq}(F, A)$ and $(F, A) \widetilde{\subseteq}(F, A)^{-1}$.

Proof. Let $(F, A)$ be a $L$-fuzzy soft group over $G$. Then $F(a)$ is a $L$ fuzzy subgroup of $G$ for all $a \in A$. Also $(F(a) \cdot F(a))(x) \leq F(a)(x)$ for all $x \in G$. Hence $(F, A) \widetilde{\odot}_{\cap}(F, A) \widetilde{\subseteq}(F, A)$. On the other hand, since $F(a)^{-1}(x)=$ $F(a)\left(x^{-1}\right) \geq F(a)(x)$, then $(F, A) \widetilde{\simeq}(F, A)^{-1}$.

Conversely, let $z=x \cdot y$ for all $x, y, z \in G$. Since $(F, A) \widetilde{\odot}_{\cap}(F, A) \widetilde{\subseteq}(F, A)$, then $F(a)(z) \geq(F(a) \cdot F(a))(z)=\bigvee_{z=x \cdot y}\{F(a)(x) \wedge F(a)(y) \mid x, y \in G\} \geq$ $F(a)(x) \wedge F(a)(y)$ for all $a \in A$. Hence $F(a)(z) \geq F(a)(x) \wedge F(a)(y)$. On the other hand, Since $(F, A) \widetilde{\subseteq}(F, A)^{-1}$, then $F(a)(x) \leq F(a)^{-1}(x)=F(a)\left(x^{1}\right)$ for all $x \in G$. Also $F(a)$ is a $L$-fuzzy subgroup of $G$ for all $a \in A$. Consequently, $(F, A)$ is a $L$-fuzzy soft group over $G$.

Theorem 24. Let $(F, A)$ be a $L$-fuzzy soft set over $G$. Then $(F, A)$ is a $L$-fuzzy soft group over $G$ if and only if $(F, A) \widetilde{\odot}_{\cap}(F, A)^{-1} \widetilde{\subseteq}(F, A)$.

Proof. It is similar to the proof of Theorem 23.

Definition 25. Let $(F, A)$ and $(K, B)$ be two $L$-fuzzy soft sets over $G_{1}$ and $G_{2}$, respectively. Let $\phi: G_{1} \rightarrow G_{2}, \psi: A \rightarrow B$ be two functions. Then we say that the pair $(\phi, \psi)$ is a $L$-fuzzy soft function from $(F, A)$ to $(K, B)$ denoted by $(\phi, \psi):(F, A) \rightarrow(K, B)$ if $\phi(F(x))=K(\psi(x))$ for all $x \in A$. If $\phi$ and $\psi$ are injective (resp. surjective, bijective), then $(\phi, \psi)$ is said to be injective (resp. surjective, bijective).

In this definition, If $\phi$ is a group homomorphism from $G_{1}$ to $G_{2}$, then $(\phi, \psi)$ is said to be a L-fuzzy soft group homomorphism, and that $(F, A)$ is L-fuzzy soft homomorphic to $(K, B)$. The latter is denoted by $(F, A) \sim(K, B)$. If $\phi$ is an isomorphism from $G_{1}$ to $G_{2}$ and $\psi$ is a bijective mapping from $A$ onto $B$, then we say that $(\phi, \psi)$ is a L-fuzzy soft group isomorphism and that $(F, A)$ is $L$-fuzzy soft isomorphic to $(K, B)$. The latter is denoted by $(F, A) \simeq(K, B)$.

Lemma 1. Let $(F, A),(K, B)$ and $(N, C)$ be $L$-fuzzy soft sets over $G_{1}, G_{2}$ and $G_{3}$, respectively. Let $(\phi, \psi):(F, A) \rightarrow(K, B)$ and $(\varphi, \gamma):(K, B) \rightarrow(N, C)$ be two $L$-fuzzy soft functions. Then $(\varphi \circ \phi, \gamma \circ \psi):(F, A) \rightarrow(N, C)$ is a $L$-fuzzy soft function. 
Proof. Let $(\phi, \psi):(F, A) \rightarrow(G, B)$ and $(\varphi, \gamma):(G, B) \rightarrow(N, C)$ be two fuzzy soft functions. Then we can write $\phi(F(x))=K(\psi(x))$ and $\varphi(K(y))=$ $N(\gamma(y))$, for all $x \in A, y \in B$. Since $\varphi \circ \phi: G_{1} \rightarrow G_{3}$ and $\gamma \circ \psi: A \rightarrow C$, then $\varphi \circ \phi(F(x))=\varphi(\phi(F(x)))=\varphi(K(\psi(x)))=N(\gamma(\psi(x)))=N(\gamma \circ \psi(x))$ for all $x \in A$. Also $(\varphi \circ \phi, \gamma \circ \psi):(F, A) \rightarrow(N, C)$ is a $L$-fuzzy soft function.

Definition 26. Let $(F, A)$ and $(K, B)$ be two $L$-fuzzy soft sets over $G_{1}$ and $G_{2}$, respectively. Let $(\phi, \psi)$ be a $L$-fuzzy soft function from $(F, A)$ to $(K, B)$. Then,

(1) The image of $(F, A)$ under the $L$-fuzzy soft function $(\phi, \psi)$, denoted by $(\phi, \psi)(F, A)$, is a $L$-fuzzy soft set over $G_{2}$ defined by $(\phi, \psi)(F, A)=$ $(\phi(F), B)$, where

$$
\phi(F)(y)=\left\{\begin{array}{lr}
\bigvee_{\psi(x)=y} \phi(F(x)) & \text { if } \quad y \in \operatorname{Im} \psi \\
0_{G_{2}} & \text { otherwise }
\end{array}\right.
$$

for all $y \in B$.

(2) The pre-image of $(K, B)$ under the $L$-fuzzy soft function $(\phi, \psi)$, denoted by $(\phi, \psi)^{-1}(K, B)$, is the $L$-fuzzy soft set over $G_{1}$ defined by $(\phi, \psi)^{-1}(K, B)$ $=\left(\phi^{-1}(K), A\right)$, where

$$
\phi^{-1}(K)(x)=\phi^{-1}(K(\psi(x)))
$$

for all $x \in A$.

Theorem 27. Let $(F, A),(K, B)$ and $(N, C)$ be $L$-fuzzy soft sets over $G_{1}$, $G_{2}$ and $G_{3}$, respectively. Let $(\phi, \psi):(F, A) \rightarrow(K, B)$ and $(\varphi, \gamma):(K, B) \rightarrow$ $(N, C)$ be two $L$-fuzzy soft group homomorphism. Then $(\varphi \circ \phi, \gamma \circ \psi):(F, A) \rightarrow$ $(N, C)$ is a L-fuzzy soft group homomorphism.

Proof. It is straightforward.

Theorem 28. Let $(F, A)$ and $(K, B)$ be two L-fuzzy soft groups over $G_{1}$ and $G_{2}$, respectively. Let $(\phi, \psi)$ be a $L$-fuzzy soft group homomorphism from $(F, A)$ to $(K, B)$. Then,

(1) If $\phi: G_{1} \rightarrow G_{2}$ is a epimorphism of groups and $\psi$ is a bijective mapping, then $(\phi(F), B)$ is a $L$-fuzzy soft group over $G_{2}$.

(2) If $\phi: G_{1} \rightarrow G_{2}$ is a epimorphism of groups, then $\left(\phi^{-1}(K), A\right)$ is a $L$-fuzzy soft group over $G_{1}$. 
Proof. (1) Let $y \in B$. Since $\psi$ is bijective, then there exist a unique $x \in A$ such that $\psi(x)=y$. Since $F(x)$ is a $L$-fuzzy subgroup of $G_{1}$ and $\phi$ epimorphism, then $\phi(F(x))$ is a $L$-fuzzy subgroup of $G_{2}$. Also, $\phi(F)(y)=\phi(F(x))$ is a $L$-fuzzy subgroup over $G_{2}$. Hence, $(\phi(F), B)$ is a $L$-fuzzy soft group over $G_{2}$.

(2) Since $\psi(x) \in B$ for all $x \in A$ and $(K, B)$ is a $L$-fuzzy soft group over $G_{2}$, then $K(\psi(x))$ is a $L$-fuzzy subgroup of $G_{2}$ for all $x \in A$. Also, its homomorphic inverse image $\phi^{-1}(K(\psi(x)))$ is also a $L$-fuzzy subgroup of $G_{1}$ for all $x \in A$. Hence $\left(\phi^{-1}(K), A\right)$ is a $L$-fuzzy soft group over $G_{1}$.

\section{Conclusion}

In this paper we studied the algebraic properties of $L$-fuzzy soft sets in group structures. We introduced the notion of $L$-fuzzy soft groups, and gave several examples. We also investigated relations between $L$-fuzzy soft groups and soft groups. We then demonstrated that $L$-fuzzy soft groups more general concept than soft groups. Furthermore, we obtained a necessary and sufficient condition to be $L$-fuzzy soft group of a given $L$-fuzzy soft set. We hope that this work would be beneficial to extend subsequent studies.

\section{References}

[1] U. Acar, F. Koyuncu and B. Tanay, Soft sets and soft rings, Computers and Mathematics with Applications, 59 (2010), 3458-3463; doi: 10.1016/j.camwa.2010.03.034.

[2] H. Aktaş and N. Çağman, Soft sets and soft groups, Information Sciences, 177 (2007), 2726-2735; doi: 10.1016/j.ins.2006.12.008.

[3] M.I. Ali, F. Feng, X. Liu, W.K. Min and M. Shabir, On some new operations in soft set theory, Computers and Mathematics with Applications, $\mathbf{5 7}$ (2009), 1547-1553; doi: 10.1016/j.camwa.2008.11.009.

[4] A. Aygünoğlu and H. Aygün, Introduction to fuzzy soft groups, Computers and Mathematics with Applications, 58 (2009), 1279-1286; doi: 10.1016/j.camwa.2009.07.047.

[5] Y. Çelik, C. Ekiz and S. Yamak, A new view on soft rings, Hacettepe Journal of Mathematics and Statistics, 40, No. 2 (2011), 273-286. 
[6] F. Feng, Y.B. Jun and X. Zhao, Soft semirings, Computers and Mathematics with Applications, 56 (2008), 2621-2628; doi: 10.1016/j.camwa.2008.05.011.

[7] E. İnan and M.A. Öztürk, Fuzzy soft rings and fuzzy soft ideals, Neural Computing and Applications, 21 (2011); doi: 10.1007/s00521-011-0550-5.

[8] N. Jacobson, W.H. Freeman, Basic Algebra I, W.H. Freeman, San Francisco (1974).

[9] Y. Jiang, Y. Tang, Q. Chen, H. Liu and J. Tang, Interval-valued intuitionistic fuzzy soft sets and their properties, Computers and Mathematics with Applications, 60 (2010), 906-918; doi: 10.1016/j.camwa.2010.05.036.

[10] L. Jin-hang, Y. Rui-xia and Y. Bing-xue, Fuzzy Soft Sets and Fuzzy Soft Groups, Control and Decision Conference, Chinese (2008) 2626-2629; doi: 10.1109/CCDC.2008.4597801.

[11] Y.B. Jun, K.J. Lee and C.H. Park, Fuzzy soft set theory applied to BCK/BCI-algebras, Computers and Mathematics with Applications, 59 (2010), 3180-3192; doi: 10.1016/j.camwa.2010.03.004.

[12] O. Kazancı, Ş. Yılmaz and S. Yamak, Soft sets and soft BCH-algebras, Hacettepe Journal of Mathematics and Statistics, 39, No. 2 (2010), 205217.

[13] P.K. Maji, R. Biswas and A.R. Roy, Fuzzy Soft Sets, Journal of Fuzzy Mathematics, 9, No. 3 (2001), 589-602.

[14] P.K. Maji, R. Biswas and A.R. Roy, Soft set theory, Computers and Mathematics with Applications, 45 (2003), 555-562; doi: 10.1016/S08981221(03)00016-6.

[15] P. Majumdar and S.K. Samanta, Generalised fuzzy soft sets, Computers and Mathematics with Applications, 59 (2010), 1425-1432; doi: 10.1016/j.camwa.2009.12.006.

[16] D. Molodtsov, Soft set theory - first results, Computers and Mathematics with Applications, 37 (1999), 19-31; doi: 10.1016/S0898-1221(99)00056-5.

[17] J.N. Mordeson, D.S. Malik, Fuzzy Commutative Algebra, World Scientific Publishing Co, Singapure (1998). 
[18] Z. Pawlak, Rough sets, International Journal of Computer and Information Sciences Sci., 11 (1982), 341-356; doi: 10.1007/BF01001956.

[19] S. Yamak and O. Kazanc1, B. Davvaz, Soft hyperstructure, Computers and Mathematics with Applications, 62 (2011), 797-803; doi: 10.1016/j.camwa.2011.06.009.

[20] L.A. Zadeh, Fuzzy sets, Information and Control, 8 (1965), 338-353; doi: 10.1016/S0019-9958(65)90241-X. 
